Revista Arbitrada Interdisciplinaria de Ciencias de la Salud. SALUD Y VIDA

Volumen 5. Número 1. Año 5. Especial. 2021

Hecho el depósito de Ley: FA2016000010

ISSN: $2610-8038$

FUNDACIÓN KOINONIA (F.K)

Santa Ana de Coro, Venezuela.

Adisnay Rodríguez-Plasencia; Sofia Paola Sanaguano-Martínez; Manuel Andrés Cueva-Carrión

http://dx.doi.org/10.35381/s.v.v5i1.1601

\title{
Cuidados de enfermería para pacientes pediatricos con neumonia de lóbulo inferior izquierdo
}

\section{Nursing care for pediatric patients with left lower lobe pneumonia}

\author{
Adisnay Rodríguez-Plasencia \\ ua.adisnayrodriguez@uniandes.edu.ec \\ Universidad Regional Autónoma de los Andes, Ambato \\ Ecuador \\ https://orcid.org/0000-0002-5293-2817 \\ Sofia Paola Sanaguano-Martínez \\ ea.sofiapsm04@uniandes.edu.ec \\ Universidad Regional Autónoma de los Andes, Ambato \\ Ecuador \\ https://orcid.org/0000-0002-0812-5217 \\ Manuel Andrés Cueva-Carrión \\ ea.manuelacc08@uniandes.edu.ec \\ Universidad Regional Autónoma de los Andes, Ambato \\ Ecuador \\ https://orcid.org/0000-0002-4752-6179
}

Recepción: 10 de agosto 2021

Revisado: 15 de septiembre 2021

Aprobación: 15 de noviembre 2021

Publicación: 01 de diciembre 2021 


\section{Sra Editora:}

Mediante un plan de cuidados de enfermería dirigido a la atención de pacientes pediátricos que padecen neumonía de lóbulo izquierdo, desarrollado mediante estudio de caso a paciente atendido en el área de Pediatría en el Hospital Provincial General Latacunga, se crearán las condiciones para mejorar la atención, recuperación y disminuir el tiempo de estadía hospitalaria de este tipo de pacientes al momento de abandonar el área de Pediatría del servicio hospitalario y así evitar futuras complicaciones.

Por lo tanto, se elaboró el plan de cuidados de enfermería (4) para mejorar la atención de los pacientes que sufren Neumonías de Lóbulo Inferior Izquierdo en pacientes pediátricos Hospitalizados en el Servicio de Pediatría del Hospital Provincial General de Latacunga.

En el Patrón perceptivo- Control de salud, a pesar de que el paciente se encontraba sometido a procedimientos invasivos, como el cateterismo vesical y administración de medicamentos por vía intravenosa, con la intervención de enfermería se logró controlar el riesgo de infecciones, evitado así complicaciones asociadas a estas, disminuyendo la estadía hospitalaria y costos al familiar (5) (6).

En el Patrón Nutricional-Metabólico al evidenciarse una ganancia de peso insuficiente manifestada por la pérdida de apetito se realizan intervenciones encaminadas a satisfacer las necesidades del paciente, así como la monitorización del estado nutricional obteniéndose una ganancia en el peso corporal de 500gr.

Con la monitorización de los signos vitales, específicamente la temperatura corporal, asociadas a otras intervenciones de Enfermería relacionadas con la Hipertermia, se logró evitar complicaciones asociadas a este diagnóstico enfermero como son, convulsiones febriles, arritmias cardiacas o cambios celulares anómalos, que pueden interferir de manera temporal o permanente en la salud del niño. Al realizar una monitorización adecuada de las funciones respiratorias, así como la utilización de terapias respiratoria como la percusión para favorecer la expulsión de secreciones, se logró mejoras la 
mecánica ventilatoria del paciente, así como disminuir los cambios en la membrana alveolo capilar, evidenciados además por la radiografía evolutiva, realizada posterior a dicha intervención de enfermería.

\section{CONFLICTO DE INTERÉS}

Los autores declaran que no tienen conflicto de interés en la publicación del artículo.

\section{FINANCIAMIENTO}

No monetario.

\section{AGRADECIMIENTO}

A la Universidad Regional Autónoma de los Andes; por apoyar el desarrollo de la investigación.

\section{REFERENCIAS}

1. Kong LY, Longtin Y. Left-Middle-Lobe Pneumonia. N Engl J Med. 2017;377(6):e8. doi:10.1056/NEJMicm1700661

2. Thilakanathan C, Hall M, Rahman W, Magdy M, Jorgensen J. Unexplained recurrent left lower lobe pneumonia, haematemesis and splenomegaly in a 32year-old gentleman. ANZ J Surg. 2021;10.1111/ans.16976. doi:10.1111/ans.16976

3. Ramgopal S, Ivan Y, Medsinge A, Saladino RA. Pediatric Necrotizing Pneumonia: A Case Report and Review of the Literature. Pediatr Emerg Care. 2017;33(2):112115. doi:10.1097/PEC.0000000000000585

4. Tashtoush B, Memarpour R, Gonzalez J, Gleason JB, Hadeh A. Pulmonary Sequestration: A 29 Patient Case Series and Review. J Clin Diagn Res. 2015;9(12):AC05-AC8. doi:10.7860/JCDR/2015/16004.7006

5. Sun $X$, Xiao Y. Pulmonary sequestration in adult patients: a retrospective study. Eur J Cardiothorac Surg. 2015;48(2):279-282. doi:10.1093/ejcts/ezu397 
Revista Arbitrada Interdisciplinaria de Ciencias de la Salud. SALUD Y VIDA

Volumen 5. Número 1. Año 5. Especial. 2021

Hecho el depósito de Ley: FA2016000010

ISSN: 2610-8038

FUNDACIÓN KOINONIA (F.K)

Santa Ana de Coro, Venezuela.

Adisnay Rodríguez-Plasencia; Sofia Paola Sanaguano-Martínez; Manuel Andrés Cueva-Carrión

6. Ceylan KC, Batihan G, Üçvet A, Gürsoy S. Surgery in congenital lung malformations: the evolution from thoracotomy to VATS, 10-year experience in a single center. J Cardiothorac Surg. 2021;16(1):131. doi: 10.1186/s13019-021$\underline{01511-0}$

2021 por los autores. Este artículo es de acceso abierto y distribuido según los términos y condiciones de la licencia Creative Commons Atribución-NoComercial-Compartirlgual 4.0 Internacional (CC BY-NC-SA 4.0) (https://creativecommons.org/licenses/by-nc-sa/4.0/). 